\title{
Sobre el vínculo afectivo y los procesos de aprendizaje en niños de 7 a 9 años de edad
}

\section{Affectional Bonds and Learning Processes in Children Aged 7-9}

\author{
Bairon Jaramillo-Valencia \\ Sirley Largo-Tapasco \\ Laura Gómez-Monsalve \\ Universidad Católica Luis Amigó, Medellín, Colombia
}

Open Access:

ISSN: $0124-2121$

E-ISSN: $2665-2420$

ARTÍCULO RESULTADO DE

INVESTIGACIÓN
Copyright @ 2020

By Educación y Humanismo

Editor:

Patricia Martínez Barrios

Universidad Simón Bolívar

Recibido: 22-08-2019

Aceptado: $13-12-2020$

En línea desde: $15-04-2020$

DOI:

10.17081/eduhum.22.

38.355 information-collection techniques, such as interviews, observation of participants during a patchwork mat activity, workshops, and documentary analysis. Findings: This research determined the importance of affectional bonds during child development, a fact perceived in the little quality time spent by parents with their children. Furthermore, this led to learning difficulties in schoolchildren, resulting in a mediocre academic process. Discussion y Conclusion: It is argued that the breaking of the affective bond affects differently the development of boys and girls, since despite the fact that they usually overcome the break or seem to do so, in the workshop carried out it was evidenced that there are certain students who could be more affected In their learning process, this due to the different factors they have had to cope with, making the breaking of their bond more traumatic. Consequently, it was concluded from the systematic process that, the emotional bond is given through mutual sharing and quality relationships between two people.

Keywords: Affectional bond, learning process, children, education. 


\section{Introducción}

En la naturaleza del ser humano están dadas las relaciones interpersonales; comportamientos que, con el transcurso del tiempo, generan apegos, cariños, amistades; y en general, vínculos que llevan inmersos el afecto entre dos o más personas. Los vínculos afectivos son considerados formas de relación que para esta situación, y teniendo como referencia las edades base para este estudio ( 7 a 9 años de edad), se dan con frecuencia en personas que comparten cercanamente de forma especial, posibilitando así la creación de conexiones generadoras de confianza, respeto, alegría y amor. Análogamente, este sentimiento tan característico provee de soporte constante durante el desarrollo de la infancia para todo niño o niña.

En la actualidad, es común advertir que los vínculos afectivos suelen romperse de manera estrepitosa; situaciones que tienen su génesis por diferentes circunstancias, las cuales parecen ser usuales en numerosos contextos. Como consecuencia, es aquí cuando los niños y niñas-producto de dichas relaciones- suelen ser los más afectados, a pesar de no haber dado lugar a estas separaciones. El problema para esta investigación se da cuando en el contexto seleccionado, se percibe que algunas de las familias de los niños y niñas (unidades de análisis) se enfrentan a situaciones de rompimiento del vínculo afectivo, circunstancias que se justifican en las separaciones; en la muerte o el abandono de algún adulto significativo; de tal forma, se genera en el niño o niña condiciones que pueden influir en su proceso de aprendizaje, tanto a corto como a mediano o largo plazo. Por lo anterior, surge el problema de investigación, el cual se delimitó a través de la pregunta ¿Cuál es la incidencia en el proceso de aprendizaje de niños y niñas entre 7 y 9 años de la Institución Educativa Lola González, cuando se rompe el vínculo afectivo con un adulto significativo?

Por consiguiente, la propuesta de investigación se realizó con el fin de describir la incidencia que tiene la ruptura del vínculo afectivo en los procesos de aprendizaje de los niños y niñas que se encuentran entre los 7 y los 9 años de edad, además de identificar las situaciones que llevan las rupturas del vínculo afectivo con los adultos significativos que los rodean; intentando así orientar la investigación no solo con fines educativos, sino también reconociendo las etapas del proceso de aprendizaje y su respectiva incidencia en el desarrollo de los infantes.

La importancia del proyecto de investigación que sirvió como referencia para la escritura de este artículo, se centró en identificar, mostrar y presentar soluciones a las rupturas o modificaciones de los vínculos afectivos de los niños y niñas de 7 a 9 años con los adultos significativos que los rodeaban, pues se estuvo generalizando la idea en la institución, y también en la comunidad circundante, que uno de los factores principales para la alteración negativa del rendimiento académico-conductual en el desarrollo de los 
niños y niñas $-\mathrm{y}$ en general, en los cambio de los procesos de aprendizaje - se da por las modificaciones o alteraciones de los vínculos afectivos con sus pares más cercanos. Por tal motivo, este estudio permitió dar cuenta de situaciones que se vivieron a nivel institucional, y se logró avanzar en la solución de este problema, estableciendo un posible punto de partida para futuras investigaciones locales, regionales e inclusive nacionales.

Finalmente, la ruta metodológica empleada para la ejecución de este estudio se dio a través de un paradigma cualitativo y un enfoque histórico-hermenéutico; para lo cual se emplearon técnicas de recogida de la información tales como: la entrevista, el taller y el análisis documental.

\section{Marco Teórico}

En primer lugar, es imperativo definir dos concepciones necesarias para que los lectores puedan comprender, en su totalidad, lo que este artículo pone en evidencia; esto es, conocer detalladamente lo qué se entiende por vínculo afectivo y procesos de aprendizaje.

El vínculo afectivo es el nexo y coincidencia de aspectos emocionales que permiten a las personas, y del mismo modo a los niños, desarrollar sus dimensiones afectivas, generando una relación directa con aquel o aquellos a quienes se quiere, se sigue y suelen ser -de la misma forma- un ejemplo a seguir. Tal como lo menciona Calle (2013):

En toda relación de afecto surge el vínculo afectivo, es decir, una unión marcada por el cariño. Es indudable que en la pareja en la que hay cariño se da un vínculo afectivo, que es mucho más importante que la difusa actitud del compromiso ( $p$. 23).

En este sentido, se busca analizar el vínculo afectivo en conexión con la dedicación de tiempo y compañía que entrega uno o más adultos, a un niño o niña; permitiendo con ello, la creación de momentos alegres, tristes, de apoyo; y en general, tiempo de calidad, que consecuentemente generan confianza. En esta línea, Amar, Llanos y García (2004) indican que:

El vínculo afectivo es un nexo emocional recíproco y fortalecido entre un infante y la persona que lo cuida, cada uno de los cuales contribuye a la calidad de la relación. Los vínculos afectivos tienen un valor adaptativo para los bebés, garantizando que sus necesidades psicosociales y físicas sean satisfechas (p. 34).

Es por ello que se pretende estudiar que no solo se genera un vínculo con la madre, padre o hermanos, sino también con aquella persona que de dedicación pertinente; es decir, que entrega su tiempo para atender y proteger a los infantes, al igual que lo hacen 
los cuidadores. Por otra parte, sobre este aspecto se intenta vislumbrar aquellas nociones que aprende un niño o niña del adulto con el que se ha creado un vínculo afectivo; estableciendo inicialmente que de esta relación, se permite al niño aprender conceptos como el cariño, la compañía, la preocupación por el otro, el sentimiento de necesidad hacia el otro; concepciones que se dan no solo en relación con la modificación del ambiente del menor, sino que proporcionan un desarrollo cognitivo estable, pues el apoyo y el vínculo les genera seguridad. Dirksen (2004) afirma que "el aprendizaje es real cuando la información induce un cambio mental interno, caracterizado por reordenamiento de vías neurales, y puede conducir a un cambio persistente en la conducta" (p. 45). De este modo, puede llegar a ser factible observar en el niño o niña si se está logrando un desarrollo cognitivo adecuado, caracterizado por modificar su conducta y brindar unos cimientos adecuados que potencien su crecimiento personal.

Debido a la fuerza del vínculo que se origina entre un niño o niña y un adulto, se determina la afinidad que se explica con la complacencia y en el compartir de sus momentos, generando con ello un estado de gracia en el niño, el cual le permite que tenga un desarrollo emocional tranquilo, pues este tiene el apoyo, acompañamiento y protección; situaciones que, al cohesionarse, generan el vínculo afectivo. Tal como afirma Guerras (2013) haciendo referencia a la salud mental: "Los niños que tienen establecidos vínculos afectivos seguros disponen de un factor de protección muy importante desde el punto de vista emocional, ya que disponen de una gran ayuda para afrontar situaciones de crisis y para desarrollar su autoestima positiva" (p. 69). En este orden de ideas, se analiza si la unidad de los integrantes de la familia, generará un círculo mayor de estabilidad que permita al niño establecer un vínculo afectivo con varios integrantes de esta, siendo por antonomasia en innumerables momentos- de vital importancia en todas las experiencias del menor. Quevedo y Rueda (2006) mencionan al respecto que:

La familia que ha logrado crear entre sus miembros un vínculo afectivo es el mejor ambiente para esta educación en valores. Ello crea una atmósfera de respeto mutuo, de padres e hijos. Esto se logra cuando todos en familia estamos convencidos de que cada persona es única sin copia e irrepetible (p. 323).

Con relación a lo anterior, en cuanto a la pertinencia que tiene la familia en el desarrollo holístico del niño, es necesario recalcar que no solo lo tangible (como proveer alimentación, implementos escolares, ropa, etc.) llega a ser lo más importante para la formación de los infantes; ya que elementos como el acompañamientos escolar, al igual que los momentos de ocio y recreación, ofrecen al menor un soporte emocional que se concatena con su diario vivir; y de igual manera, dichas manifestaciones afectivas las refleja hacia los demás. Al respecto, Antolinez, Triviño y Bolívar (2018) declaran que: "la familia tiene funciones insustituibles como son: el cuidado y educación de los hijos y el apoyo afectivo entre adultos unidos por vínculo afectivo. Por ello se reconoce que la familia tiene cuatro funciones fundamentales: sexual, económica, reproductiva y educativa" (p. 39). 
Dado que este trabajo tiene la intención de brindar ayuda los padres y/o madres de los menores que participaron en este estudio, se pretende lograr entender si estos permiten a los infantes, una cohesión adecuada con su entorno y entienden que -como establecen Largacha y Gómez (2015) "El aprendizaje es un proceso que exige la práctica, la repetición, el ensayo y el error. Exige, por supuesto, la reflexión sobre el error, antes que su castigo o su sanción" (p. 13). Situaciones algunas veces complejas de por sí, por el poco entendimiento que los padres tienen sobre las diversas formas de aprendizaje en los niños y niñas.

Precisamente sobre esa diversidad en los procesos de aprendizaje, los padres de familia o acudientes deben ser conscientes de los distintos modos de aprendizajes; entre los cuales se rescata el auto-estructurante. Un proceder que - no entendido como delegar todas las responsabilidades procedimentales al niño- se caracteriza por permitir que el aprendiz construya su propio conocimiento a partir de la guía intermitente de los adultos o profesores:

El aprendizaje autónomo es un proceso educativo que busca desarrollar una serie de habilidades y técnicas en el aprendiente que le permita dinamizar y monitorear su proceso de aprendizaje, debe el aprendiente dirigir, controlar, regular y evaluar su proceso de aprendizaje, además de ser capaz de construir por sí mismo y conscientemente, su conocimiento (Mejía, 2009, p. 123-124).

En consonancia con los procesos autónomos de aprendizaje, se trae a colación un complemento que amplía la visión de este concepto, ya que -como se mencionó anteriormente - no significa delegar todas las responsabilidades al niño para que lleve a cabo sus deberes académicos sin ayuda externa alguna. Por lo contrario, el aprendizaje autónomo debe nutrirse paulatinamente; un actuar que el adulto a cargo o docente debe movilizar con sensibilidad y no súbitamente, pues el impacto y la angustia del infante frente a un proceder nuevo que debe incorporar en su comportamiento, puede llevarlo a la interrupción voluntaria de sus labores académicas, hasta el punto de tener apatía por las actividades de clase o tareas que requieran mucho esfuerzo individual. En lo tocante al trabajo autónomo en relación a los procesos educativos, Valdivieso (2018) ratifica que:

La educación, para ser pertinente, debe preparar a los ciudadanos del futuro para un proceso de aprendizaje, continuo y autónomo, a lo largo de su vida; además, debe promover el trabajo multidisciplinario, la integración apropiada y crítica de las tecnologías, el dominio de otros idiomas, incentivar la disciplina, la dedicación, la fijación de metas y el sentido crítico (p. 196).

Por otra parte, cuando se hace referencia a procesos de aprendizaje, no se puede inexorablemente soslayar la implementación de la tecnología en las dinámicas de enseñanza-aprendizaje. Las TIC, y en especial los aparatos electrónicos, controlados eficientemente por los adultos, pueden ofrecer al niño-aprendiz una forma de complementar su proceso de búsqueda, lectura y percepción de contenido audiovisual. Análogamente, es necesario que los acudientes o padres de familia sean los que controlen 
en sus niños tanto el tiempo de uso de las computadoras, celulares o tabletas, como los programas o aplicaciones que allí se consignan, puesto que -al igual que en la realidad objetual - se debe acompañar a los infantes en ese descubrimiento de la vida para que todo no dependa de una experimentación primaria y descontrolada de ellos para con la realidad:

El grado de incorporación tecnológica a la educación, puede afirmarse que también ha fortalecido los modelos pedagógicos por los cuales se transfiere y evalúa el conocimiento en los estudiantes, este principio genera espacios diferentes de aprendizaje caracterizados por fomentar la habilidad crítica, el pensamiento disruptivo y el trabajo colaborativo (Melamed y Miranda, 2016, p. 181).

Por último, es importante mencionar en este apartado, el vínculo afectivo que tienen los niños y niñas con un adulto, y el proceso que realizan los educadores; quienes buscan potencializar el conocimiento de sus educandos, el cual depende -en muchas ocasionesde la estabilidad emocional que le brinden. Con relación a lo anterior, Villao y Espinoza (2016) establecen de manera acertada que:

El aprendizaje es un proceso en el cual interviene el docente como mediador y facilitador del conocimiento y el estudiante es quien se apropia de la ciencia a través de las diversas estrategias, condiciones que se dan junto a otros factores: cognitivo, afectivo, motivacional, social, cultural e institucional (p. 35).

De esta forma, se intentará dar cuenta del vínculo afectivo y cómo este puede afectar los procesos de aprendizaje de los niños y niñas de la INSTITUCIÓN EDUCATIVA LOLA GONZÁLEZ.

\section{Método}

El proyecto de investigación que sirvió como referencia para la redacción de este texto, aborda el paradigma cualitativo como referencia metodológica, ya que se enfatiza en la comprensión de las situaciones que desarrollan las unidades de análisis en sus respectivos contextos (Cardona et al., 2019). Del mismo modo, la subjetividad es tenida en cuenta para entender la construcción que se desarrolla en la realidad en la que viven las personas; en este sentido, Monterrubio, Sosa y Osorio (2018) refieren que: "La investigación cualitativa no privilegia el número de datos, como la cuantitativa, sino la selección de informantes estratégicos y la profundidad de las respuestas, así como la contextualización de las mismas, lo que aporta valiosos insumos para el análisis" (p. 109). El paradigma cualitativo permite conocer cuáles son las incidencias en el proceso educativo de los niños y niñas que se encuentran en diferentes contextos familiares; principalmente, enfocados en aquellos niños en los que se está evidenciando ruptura del vínculo afectivo a causa de separación, abandono y/o fallecimiento de alguno de sus padres. Es de gran importancia para esta investigación, el uso de la perspectiva anteriormente mencionada, debido a que permite extraer datos textuales de la realidad. Además, posibilita identificar la realidad familiar y educativa que vivencia los niños y niñas. 
Por otro lado, la investigación apunta hacia un enfoque histórico-hermenéutico, el cual asocia la realidad como una obra humana, la cual posee diferentes factores que van ligados al ser y a la construcción de la conducta del niño y la niña. Esta perspectiva se basa no solo en la realidad, sino que su aplicación práctica se genera en los niños, intentando extraer de ellos todas esas conductas, emociones y experiencias. Con relación a este tema, se indica la siguiente definición:

El interés histórico-hermenéutico concibe la realidad como una construcción humana, histórica, dinámica y compleja, que requiere ser leída, comprendida, problematizada e interpretada a partir de una reconstrucción articulada y con sentido del devenir histórico de los sujetos; de este modo se podrán poner en evidencia las maneras como se configuran redes y rupturas, vínculos y conflictividades, encuentros y desencuentros (García et al., 2002, p. 56).

Para este proceder en particular, es oportuno trabajar bajo el tipo de investigación denominado 'Estudio de Caso', el cual se considera funcional tanto para estudios descriptivos como explicativos, por su peculiaridad en el análisis de fenómenos particulares que se dan en un ambiente particular:

Las investigaciones realizadas a través del método de estudio de caso pueden ser: descriptivas, si lo que se pretende es identificar y describir los distintos factores que ejercen influencia en el fenómeno estudiado, y exploratorias, si a través de las mismas se pretende conseguir un acercamiento entre las teorías inscritas en el marco teórico y la realidad objeto de estudio (Carazo, 2006, p. 171).

En efecto, este modo de concebir la investigación brinda la posibilidad de realizarse desde uno o varios casos; comprendidos desde análisis teóricos, que cumplen la función de dar cuenta de cómo y porqué surgen dichas problemáticas. Esta perspectiva investigativa es precisa para el estudio, ya que trata sobre una problemática social y argumenta la posibilidad de obtener, mediante casos reales, los datos necesarios para llegar a una conclusión sobre la problemática; asimismo, intenta identificar las razones y las consecuencias del mismo. Precisamente, para lo cual será necesario utilizar a la realidad que viven los niños y niñas en caso de la separación con un adulto significativo.

Para la realización de dicha investigación, se contó con la participación de la Universidad Católica Luis Amigó y la INSTITUCIÓN EDUCATIVA LOLA GONZÁLEZ; del mismo modo, se aplicaron las técnicas de recolección en este segundo lugar, el cual está ubicado en la ciudad de Medellín-Colombia, barrio Santa Lucía. Se trabajó con una muestra de 16 estudiantes (niños y niñas de segundo grado) en la jornada de la mañana, cuyas edades oscilaban entre los 7 años y los 9 años. Del mismo modo, tenían la singularidad de vivir en hogares constituidos, ya sea por familias nucleares, extensas o monoparentales; y como característica principal, la falta de un adulto significativo en estos espacios. A su vez, participaron 16 padres de familia, acudientes de los niños y niñas anteriormente mencionados.

Teniendo en cuenta que la estrategia metodológica es el estudio de casos, se aplicaron 
diversas técnicas de recogida de la información, tales como: observación participante, entrevista semi-estructurada, análisis documental y talleres de padres de familia a través de una actividad de colcha de retazos. Los anteriores procederes investigativos permitieron llegar a la información de manera directa, logrando así recolectar todo lo necesario para alcanzar los objetivos propuestos.

La información se recolectó con la ayuda de matrices de transcripción y codificación, las cuales permitieron relacionar y discernir lo intrínsecamente tocante a las categorías del estudio (proceso de aprendizaje, vínculo afectivo y adulto significativo). A su vez, se utilizaron matrices categoriales para seleccionar los resultados de cada técnica en relación a las categorías en cuestión; y de esta manera, se pudo depurar la información-exceso que no apuntaban a la investigación per se. Esta misma segunda matriz de análisis, posibilitó relacionar las categorías con los objetivos específicos; información que fue confrontada con investigaciones que trabajaban temas similares.

\section{Resultados}

Según los resultados arrojados por la investigación realizada, se encontraron múltiples hallazgos relacionados a la incidencia del vínculo afectivo en el proceso de aprendizaje. En esta sección se desarrolla una concatenación, confrontando resultados análogos de otras investigaciones con los propios de este estudio, trayendo a colación una serie de bibliografías para soportar los argumentos expuestos. Por lo cual, se da respuesta a los objetivos relacionados a las categorías establecidas en esta investigación.

En primer lugar, del análisis documental, se observó que la mayoría de los participantes - según sus calificaciones - llevaban un proceso regular que era reiterativo desde primero hasta el grado que cursan actualmente (segundo de primaria), el cual oscilaba mayormente entre calificaciones que iban de 2.0 hasta 3.3. Se exhibe entonces, la gráfica que muestra el promedio académico de los estudiantes participantes de este estudio durante el tiempo en el cual padecían un proceso de divorcio en sus hogares; aspecto influyente, que se discutirá más adelante, entre la relación afectiva y los procesos de aprendizaje: 
Gráfico 1. Promedio de calificaciones en los estudiantes a lo largo del proceso-divorcio, cuyos protagonistas fueron los padres de familia

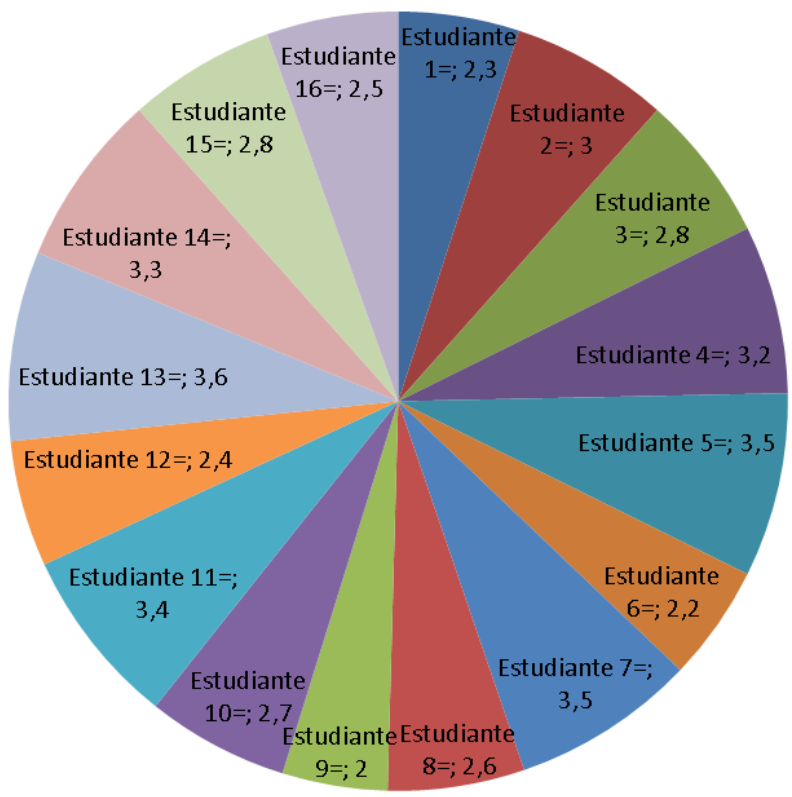

Fuente: Elaboración propia (2019).

Con el fin de conocer si el bajo rendimiento de los participantes se debió en parte a la situación del divorcio de sus padres, más adelante se presentará una gráfica que describe la respuesta académica de los niños y niñas, meses posteriores a estos momentos de impacto para sus respectivas vidas. Asimismo, sobre la información evidenciada en la anterior figura, se puede comprender la realidad en lo concerniente a la influencia que pudo tener el divorcio de los padres en el rendimiento académico de los niños y niñas, debido a que las calificaciones develan un bajo promedio en las notas (en su mayoría de 2.2 a 2.8), y un grupo minoritario entre 3.0 y 3.6. Aunque lo fundamental de la problemática no es de corte cognitivo, es eminentemente improbable aislar un fenómeno de este calibre hacia los elementos académicos, puesto que cuando el sujeto - sin importar su edad- está expuesto en reiteradas ocasiones a una serie de eventos traumáticos desde el punto de vista emocional, otros aspectos de su vida suelen ser permeados en diferentes proporciones. Por lo anterior, en una investigación denominada Consecuencias del divorcio-separación en niños de edad escolar y actitudes asumidas por los padres, ejecutada por Nuñez, Pérez y Castro (2017), se encontró que:

También se vio afectada el área escolar por presencia de afectaciones en la atención, memorización, disminución en el rendimiento académico, indisciplina y pérdida del interés escolar. Los resultados obtenidos en las técnicas aplicadas con las maestras fueron similares a las identificadas en los niños estudiados por el investigador donde la de mayor afectación resultó ser la emocional en 13 de los escolares (81,3\%), donde la tristeza y angustia prevalecieron (p.15).

Para reforzar este aspecto del impacto que tiene el vínculo afectivo de los padres que se divorcian en los procesos de aprendizaje en los niños-aprendices, se presenta otra 
investigación que trabajó el tema de la escolaridad en los infantes a través del fenómeno del divorcio en el hogar:

Cuando se produce un proceso de divorcio el aprendizaje del menor se puede alterar porque el acontecimiento interfiere en los patrones de estudio. Los menores cuyos padres se han separado alcanzan niveles de graduación más bajos y presentan un mayor fracaso escolar y ausentismo que los que provienen de familias intactas (Rodas, 2016, p. 54-55).

Partiendo de que la investigación anteriormente nombrada se encuentra basada en las consecuencias que enfrentan los niños a causa del divorcio, y realizado el análisis de la población estudiada, esto da cuenta que los niños y niñas de esta investigación han enfrentado situaciones en las que se ha visto afectado su vínculo afectivo con alguno de sus adultos significativos; y por consiguiente, más adelante se ahondará sobre este tema en testimonios dados en las entrevistas por parte de los estudiantes.

Conectando con lo anterior, se distingue que el bajo rendimiento académico se ve estrechamente relacionado a factores como la ruptura del vínculo afectivo, en donde los niños y niñas se enfrentan a circunstancias que afectan directamente sus emociones, generando así un estancamiento durante el proceso de aprendizaje, y afectando la atención y el proceso actitudinal evidenciado en la pérdida de interés por el acto de aprender. Cabe destacar que esto desencadena, en la mayoría de los casos, en la deserción escolar, aunque también esta investigación, arrojó datos de un caso particular, en el que una estudiante es de las más destacadas del grado, y no presentó ningún tipo de afectación en su proceso académico; es un hecho atípico aislado a la tendencia de los hallazgos. Sobre este aspecto, Pérez (2019) determina que:

El divorcio, la pérdida de uno y otro de los padres, precipita angustia, dolor o depresión, que a menudo se exterioriza en actos agresivos y conducta destructiva. En algunos casos el niño adopta actitudes pasivas, también presenta frecuentemente sentimientos de culpabilidad si cree ser la causa de la derrota familiar y tiene reacciones auto punitivas. Las reacciones más usuales son de orden depresivo y van acompañadas con la falta de interés hacia la culminación de sus estudios (p. 113).

Por otra parte, a producto del análisis documental en el rendimiento de los estudiantes meses posteriores al proceso de divorcio de los padres, se aprecia en la segunda figura un cambio positivo en lo académico. 
Gráfico 2. Promedio de calificaciones en los estudiantes, meses posteriores al proceso-divorcio, cuyos protagonistas fueron los padres de familia

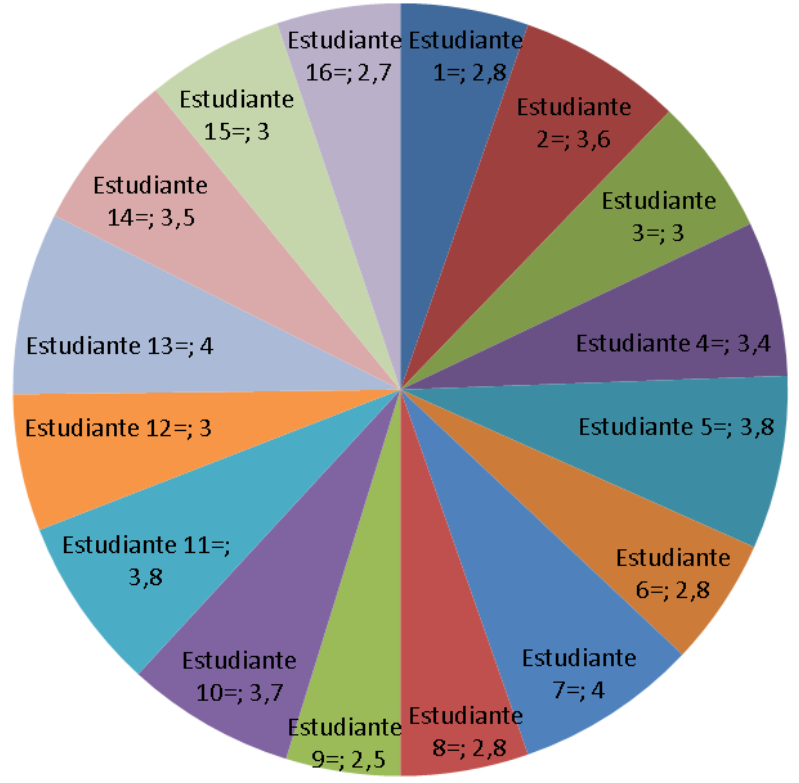

Fuente: Elaboración propia (2019).

Como se puede apreciar, la revisión de los documentos devela, si bien no muy exponencialmente, una diferencia significativa comparando las calificaciones que representaban el proceso académico de los niños y niñas durante la experiencia del divorcio de sus padres. En este sentido, gran parte de estos participantes estuvieron, en esta ocasión, en un nivel considerado "Aceptable" y "Bueno", representado en notas que oscilaban entre 3.0 y 3.6, además de 3.7 y 4.0. Por otra parte, al margen de esta tendencia, cuatro estudiantes siguieron puntuando un promedio considerado "Bajo". Sin embargo, comparando estas calificaciones con la primera medición, el porcentaje no llega a considerarse negativo en sí, puesto que está más cerca a lo aceptable, puntuaciones de 2.7 a 2.8. Como resultado, se demuestra que tiempo posterior al evento traumático de la separación por parte de los padres, los estudiantes pueden llegar a mostrar mejoras desde el punto de vista académico; esta premisa toma forma con los resultados expuesto en este estudio, concluyendo bajo esta evidencia que el divorcio influye en el bajo rendimiento de los estudiantes. Teniendo como referencia lo anteriormente enunciado, el divorcio parece traer consigo un relativo distanciamiento de las figuras paternas con el niño o niña, acentuado desde el paso de la infancia hacia la adolescencia, "donde una de sus principales características es la distancia que los niños y niñas empiezan a tomar de sus padres y de los referentes de autoridad" (Pulido, 2010, p. 152). Esta afirmación, proviene de un estudio que puso de manifiesto aspectos de la cultura escolar, en donde niños y jóvenes experimentan diversos elementos constitutivos de esas edades, tales como el bullying, la violencia, el acompañamiento de los padres, entre otros.

Por otra parte, en lo que respecta a dar cuenta del poco conocimiento que tienen los 
padres para con sus hijos, y la dificultad al momento de identificar las cualidades que evidencian en estos, se pudo observar en la aplicación de la técnica Taller con los padres, al momento de desarrollar la actividad denominada ¿Cuánto conoces a tu hijo?, que estos presentaban dificultades al hablar sobre las cualidades de sus hijos, expresando angustia por la realización de la misma: "Ojo papás, estamos haciendo todos el oso aquí delante de las profes" (Acudiente 15, comunicación personal, 10 de marzo, 2018); "Hay actitudes de los niños que solo las expresan cuando están con los amiguitos, uno como padre solo conoce lo fundamental" (Acudiente13, comunicación personal, 10 de marzo, 2018); "Todos como mamás y papás conocemos a nuestros niños, pero si les dedicáramos más tiempo, creo que los conoceríamos mejor" (Acudiente 2, comunicación personal, 10 de marzo, 2018). Sobre este tema, se suele poner toda la obligación en la escuela; en este sentido, los maestros y las maestras dan reporte a los padres de familia sobre el proceso de los niños, y es esta la forma en que usualmente ellos conocen plenamente a sus hijos, de segunda mano por parte de los docentes, los cuales deben apelar a múltiples elementos; entre estos la lúdica, actitud que moviliza las sensibilidades más humanas de los aprendices desde el sector educativo:

En lo concerniente a la actitud lúdica, se debe definir que es, si no el más importante, uno de los aspectos más significativos en cuanto a formación de maestras de educación inicial se refiere, ya que es la forma en cómo se le va a llegar al público infantil, maneras llamativas y sugerentes para captar la atención de esta población y posibilitar el aprendizaje (Quintero y Valencia, 2018, p. 90).

Posterior a esto, en la ejecución de la observación participante, a través de la realización de la Colcha de Retazos se encontró, según la interpretación de los dibujos realizados por los niños y niñas, que muchos de los adultos significativos evidenciaban ausencia en las muestras de cariño y afecto para con los estudiantes; verbigracia, muchas representaciones daban cuenta, simbólicamente, que los niños eran receptores de dichos sentimientos por parte de abuelos, abuelas, tíos, tías, amigos y mascotas; dato preocupante; dibujos dicentes, en los que situaban a los padres en segundo y tercer lugar. Con relación a lo anterior, Pérez y Arrazola (2013) en su investigación Vínculo afectivo en la relación parento-filial como factor de calidad de vida, manifiestan que:

La distribución del uso del tiempo para la realización de las actividades cotidianas es otra limitante encontrada en este estudio, el $41,8 \%$ de los padres concede poca importancia a este aspecto, son pocos los padres o cuidadores que disponen de espacios para compartir algunas vivencias anécdotas o simplemente para el diálogo intrafamiliar (p. 30).

De este modo, se da cuenta de la falta de atención que algunas veces los adultos significativos otorgan al acompañamiento que brindan a los niños; en el que, en su mayoría, son pocos los que comparten calidad de tiempo, esto se refiere a propiciar momentos de apertura al diálogo y a la expresión de sus emociones, necesidades y pensamientos; favoreciendo así el fortalecimiento de las relaciones afectivas entre padres e hijos y/o adultos significativos. En lo concerniente al tema del acompañamiento de los 
padres, Orjuela y Morera, (2016) establecen lo siguiente:

Como antecedentes al tema del acompañamiento de los padres en el proceso escolar de sus hijos se encuentran diversas concepciones importantes en las cuales se reflexiona como a través del tiempo se ha ido dejando el tema de la educación solo a las instituciones educativas, varios son los factores que inciden en el rendimiento escolar (p. 15).

Consecuentemente, se denotan diferentes conflictos familiares, en los cuales los niños se encuentran involucrados por parte de los adultos, aquí se expresan las diferencias entre sus familiares; como en el caso de una estudiante, quien lo hace saber en la entrevista semi-estructurada, técnica aplicada en esta investigación: "cuando yo tenía 6 años, mi mamita se fue de la casa con mi primo, pues él dice que mi mama lo ofende mucho, pero eso es mentira; mi papá para conseguirse otra novia" (Estudiante 7, comunicación personal, 10 de abril, 2018). De acuerdo con esto, se considera pertinente resaltar este aspecto, y es así que se perciben las afectaciones que sufren los niños en situaciones de conflictos intrafamiliares, en las que se ven directamente relacionados, y se dan por enterados de las diferencias entre sus padres y/o familiares; afectando así sus emociones. En este orden de ideas, una estudiante manifestó lo siguiente: "Cuando mi papá se va de viaje, mi mamá sale con las amigas y llega tarde a la casa, luego cuando él viene se da cuenta y se ponen a pelear" (Estudiante 14, comunicación personal, 10 de abril, 2018). Asimismo, se evidencia que la ruptura del vínculo afectivo, en algunas ocasiones se da a consecuencia del divorcio, abandono y drogadicción, en donde los niños expresan sentimientos de tristeza y melancolía. En su investigación Crisis en la infancia: ¿qué piensan, sienten y dicen los niños sobre la separación de sus padres?, Cifuente y Milicic (2010) afirman que:

La dinámica de conflicto intenso y sostenido que se produce entre los padres, aún después de producida la separación. Esta experiencia afecta negativamente a los hijo/os quienes a menudo se ven envueltos en las peleas de la ex pareja. En consecuencia, los niños manifiestan, a través de conductas y síntomas, la tensión permanente en la que viven (p. 471).

Es completamente incuestionable que los niños durante su crecimiento $-\mathrm{y}$ en relación al compartir con los adultos que los rodean, en especial con sus padres y madresgeneran vínculos; unos más débiles que otros, siendo los más fuertes aquellos que se perciben como significativos para el menor, tal como fue expuesto precedentemente. Asimismo, es fundamental entender que los vínculos significativos que crean los niños con un adulto, suelen darse en razón de las relaciones amistosas y mayormente amorosas que existen entre estos. Por ejemplo, entre cónyuges, compañeros permanentes o novios; permitiendo esto, el compartir del diario vivir entre las personas que generan la conexión afectiva. El problema de estos vínculos significativos surge cuando se rompen situaciones que se dan a causa de los divorcios o separaciones de sus padres, por razones de drogadicción o por abandonos entre los mismos, momentos en los cuales los niños no tienen ningún significante o son el motivo de que esto ocurra. Al romperse el vínculo, 
suelen verse afectados los procesos escolares de los menores, causando diferentes consecuencias negativas en los niños y niñas, dentro de las que se pueden establecer, por ejemplo, la deserción escolar, afectando con ello no solo su crecimiento cognitivo sino también el psicosocial: "Para un hijo, la separación de sus padres o el fallecimiento de alguno de ellos puede venir acompañado de una serie de procesos psicológicos que incidan sobre su probabilidad de deserción" (Ossa, 2017, p. 4).

Por otro lado, la ruptura del vínculo, a veces, se da por factores externos o sesgado por miembros de la familia; como ejemplo se expone lo siguiente: Frente a la pregunta en la entrevista semi-estructurada ¿Te han separado de alguien importante para tu vida? Algunos participantes compartieron los siguientes testimonios: "Mi papá, pero mi mamá dice que él no es importante" (Estudiante 3, comunicación personal, 10 de abril, 2018).Otro escolar entrevistado nos muestra lo siguiente: "Mi papá y sentí tristeza, porque él tomó drogas y entonces uno no puede salir con alguien drogadicto" (Estudiante 1, comunicación personal, 10 de abril, 2018). Con base a lo anteriormente expuesto, una investigación llevada a cabo durante los años 2012 y 2013, la cual se enfatizó en el comportamiento familiar y los lazos que se establecen entre sus miembros, evidenció entre sus resultados lo siguiente:

Se resalta que el mayor tiempo compartido en familia está representado en un $28,3 \%$, ofreciendo acompañamiento durante todo el día, aspecto que es significativo en la medida que posibilita el fortalecimiento de los lazos familiares, puesto que se cuenta con suficiente tiempo para compartir y dialogar, lo que permite conocer aspectos determinantes de las personalidad, el sentir y querer de los niños/as, para de este modo orientarlos, apoyarlos y, también si se requiere, desaprobar lo que no sea conveniente para ellos (Pérez y Arrazola, 2013, p. 26).

De acuerdo a la revisión de la literatura, se evidencia que de la dedicación, entrega y consagración, que se da con el tiempo que se proporciona a los niños y niñas, permite que se genere una relación de empatía familiar, la cual se verá fortalecida por el aumento de la confianza mutua entre el adulto y el niño o niña, ya que permite - con el paso del tiempo- el afianzamiento y la comprensión mutua, el conocimiento del otro y la búsqueda de la felicidad recíproca. Estas situaciones relacionales, cuando se fortalecen adecuadamente, son la génesis del vínculo afectivo que se crea entre el niño o niña y el adulto significativo, siendo ese agente cercano al menor, el que ha volcado su tiempo y su atención con el afán de lograr que este se encuentre en buenas condiciones y tenga una vida feliz.

En el caso concreto, es decir, en el de los niños y niñas entrevistados a lo largo de este proyecto de investigación, se pudo conocer que estos se encontraban en una situación posterior al rompimiento del vínculo afectivo, a causa de la separación que han vivido de alguna persona, ya sea a causa de drogadicción, divorcio o abandono; situación que en ocasiones - como queda demostrado en este texto- es un detonante generador de sentimientos, dolores, malestares y manifestaciones en los niños y niñas, quienes dependiendo de su capacidad o resiliencia, se verán menor o mayormente afectados. 
Resulta importante mencionar que en muchas ocasiones, los padres o adultos no tienen en cuenta los sentires o sentimientos de los niños y niñas, pues toman decisiones que son de vital importancia y que modifican las relaciones personales del menor con su entorno, generando con ello, el rompimiento de vínculos afectivos que posibilitan estabilidad y felicidad en los niños y niñas: "Los niños no solo cambian su forma de vivir dentro del hogar, sino que esta ruptura familiar también les va a cambiar su estilo de vida dentro del colegio y en otras actividades diarias fuera del hogar" (Jaramillo, 2016, p. 40).

Es de resaltar que en este estudio los adultos parecieron ignorar, de alguna forma, los sentimientos del menor cuando se tomaban decisiones, y desconocían además lo que puede sentir el infante a raíz de estos sucesos; y peor aún, estos parecieron soslayar el daño interno que sufren aquellos, quienes por su inmadurez o poca experiencia -e incluso debido a la reducida atención que se les presta-, no expresan de manera adecuada sus padecimientos; sentimientos o emociones experimentadas con la ruptura del vínculo afectivo, pues es esa persona perdida, quien en múltiples ocasiones, escuchaba al menor y estaba al tanto de todo lo que podía ocurrirle.

\section{Discusión y Conclusiones}

Se puede concluir que al iniciar el trabajo práctico, luego de toda la fundamentación, planeación y estudio previo, se tenían expectativas muy altas, ya que se creía que la participación de los padres y madres iba a ser extensa, debido a que los contextos y temas que se estudiarían en los talleres programados, ayudarían a mejorar el entendimiento padre- madre y niño, sin embargo, previa la citación a la Escuela de Padres que se hizo con el tiempo oportuno y por medio de la institución educativa - quien fue fundamental por la ayuda y diligencia brindada-, se evidenció el poco interés de los padres y madres en estos temas, ya que solo acudieron el mismo número de estudiantes (muestra) a la cita, lo que consecuentemente permite entender el poco interés de los citados en los asuntos que les puede educar y les permite entender a sus hijos.

Se percibe que un vínculo afectivo se construye por medio del compartir mutuo, de la cercanía diaria, de la atención, la dedicación y el tiempo; lo que permite que los niños construyan lazos con otra persona, mediante la realización de diferentes actividades que, consecuentemente, conllevan a que ellos aprendan a confiar y a querer al adulto; generando con ello, un nexo que es de vital importancia para el desarrollo y el aprendizaje.

Se discurre que el rompimiento del vínculo afectivo afecta de manera diferente el desarrollo de los niños y niñas, ya que a pesar de que suelen superar el rompimiento o parecen hacerlo, se evidenció en el taller que hay ciertos estudiantes que podrían tener una mayor afectación en su proceso de aprendizaje, debido a los diferentes factores que han tenido que sobrellevar, ya que el rompimiento de su vínculo ha sido más traumático. 
Sin embargo, estos tienen un mejor desarrollo que aquellos en los que el rompimiento del vínculo ha sido menos traumático.

Se puede evidenciar que de las unidades de análisis (niños y niñas) - quienes sufrieron el rompimiento del vínculo afectivo con algún adulto, y que tienen un mejor proceso de aprendizaje - son los que han tenido que superar una mayor cantidad de adversidades, en razón de que esto les ha permitido desarrollar habilidades en las que han tenido que tolerar diferentes momentos negativos.

En general, fue evidente en la presente investigación -a pesar de las diferentes capacidades de cada niño, siendo algunos más tolerantes al rompimiento del vínculo afectivo que han padecido- que todos manifiestan dificultades por la ruptura, ya que esto conlleva a la disminución de la atención que se les presta, pues el vínculo desaparece abruptamente y ya no tienen a esta persona a su lado.

Al finalizar, se pudo entender que en ocasiones los padres no tienen el pleno conocimiento de lo que experimentan sus hijos, de lo que sufren con el rompimiento del vínculo afectivo, y sus afectaciones a los procesos del aprendizaje que consecuentemente viven, circunstancias que tiene el menor afectado en su mente, pero que no tiene la forma o no conoce el medio para expresarlo. Asimismo, una de las causas más relevantes en la ruptura del vínculo afectivo de los niños con un adulto significativo, es el divorcio de los padres, aunque se debe reconocer que también se da por otros factores como el abandono, la drogadicción y los conflictos intrafamiliares.

\section{Referencias}

Amar, J. A., Llanos, R. A., y García, D. T. (2004). Desarrollo infantil y construcción del mundo social. Barranquilla: Ediciones Uninorte.

Antolinez, M. E. C., Triviño, N. L. A. \& Bolívar, D. M. R. (2018). Significados de la paternidad en niños y niñas de hogares con jefatura femenina. Educación y Humanismo, 20(35), 36-57. Doi: https://doi.org/10.17081/eduhum.20.35.3044

Calle, R. (2013). El arte de la pareja: saber asir, saber soltar. Madrid: KAILAS Editorial.

Carazo, C. (2006). El método de estudio de caso Estrategia metodológica de la investigación científica. Pensamiento y gestión, (20), 165-193.

Cardona, M. N., Fernández, Y. S., Jiménez, H. S., Jaramillo, V. B., Cifuentes, L. M., y Bustamente, R. L. (2019). ¿Enseñar inglés a través de lenguajes expresivos? representaciones emergentes a partir de una reflexión investigativa. En M., Córdoba (Ed.) Gestión del conocimiento perspectiva multidisciplinaria (pp. 373- 
386). Zulia, Venezuela: Fondo Editorial Universitario de la Universidad Nacional Experimental Sur del Lago "Jesús María Smprúm". Recuperado de https://www.academia.edu/40998021/_Ense\%C3\%B1ar_ingl\%C3\%A9s_a_trav\%C 3\%A9s_de_lenguajes_expresivos_Representaciones_emergentes_a_partir_de_una _reflexi\%C3\%B3n_investigativa

Cifuente, O., y Milicic, N. (2010). Crisis en la infancia: ¿qué piensan, sienten y dicen los niños sobre la separación de sus padres? Universitas Psychologica, 11(2). 469-480. DOI: https://doi.org/10.11144/Javeriana.upsy11-2.cips

Dirksen, L. H. (2004). Enfermería Medicoquirúrgica: Valoración y cuidados de problemas clínicos. Madrid: Elsevier.

García, B., González, S., Quiroz, A., Velásquez, A., y Ghiso, A. M. (2002). Técnicas interactivas para la investigación social cualitativa. Medellín: Editorial Funlam.

Guerras, S. P. (2013). La salud emocional en la infancia: componentes y estrategias de actuación en las escuelas. Madrid: NARCEA.

Jaramillo, T. P. (2016). Las experiencias de niños entre diez a doce años que han atravesado por el divorcio de sus padres (Tesis de Pregrado). Universidad San Francisco de Quito USFQ, Quito, Ecuador. Recuperado de http://repositorio.usfq.edu.ec/handle/23000/5472

Largacha, E. E., y Gómez, A. F. (2015). Aprender a escribir en la universidad. Bogotá: Universidad de los Andes.

Mejía, E. (2009). Un cambio de actitud a partir del aprendizaje autónomo para potenciar el desarrollo de la inteligencia. Educación y Humanismo, 11(17), 118-125. Recuperado

de: http://revistas.unisimon.edu.co/index.php/educacion/article/view/2123

Melamed, E., y Miranda, C. (2016). Transferencia tecnológica en la educación. Educación y Humanismo, 18(31), 180-185. Doi: https://doi.org/10.17081/eduhum.18.31.2357

Monterrubio, C., Sosa, P., y Osorio, M. (2018). Impactos del turismo residencial percibidos por la población local: una aproximación cualitativa desde la teoría del intercambio social. LiminaR. Estudios Sociales y Humanísticos, 16(1), 103-118. DOI: https://doi.org/10.29043/liminar.v16i1.567

Nuñez, M., Pérez, C. C., y Castro, P. M. (2017).Consecuencias del divorcio-separación en 
niños de edad escolar y actitudes asumidas por los padres. Revista Cubana de Medicina General Integral, 33(3) ,296-309.

Orjuela, E., y Morera, Á. (2016). Cartilla lúdica para mejorar el acompañamiento escolar de los padres a los estudiantes de grado cuarto en el colegio Grancolombiano IED. (Tesis de Especialización). Fundación Universitaria los Libertadores, Bogotá, Colombia. Recuperado de https://repository.libertadores.edu.co/handle/11371/845

Ossa, G. M. I. (2017). El efecto de tener una familia monoparental en la deserción escolar: evidencia para Chile. (Tesis de Maestría). Pontificia Universidad Católica de Chile, Santiago, Chile. Recuperado de https://repositorio.uc.cl/handle/11534/21268

Pérez, B., y Arrazola, E.T. (2013). Vínculo afectivo en la relación parento-filial como factor de calidad de vida, Tendencias y retos, 18(1), 17-32.

Pérez, P. M. R. (2019). Factores que influyen en la deserción escolar en instituciones de gestión oficial del turno noche. Revista Científica de la Facultad de FilosofíaUNA, 8(1), 106-119.

Pulido, L. E. C. (2010). Cultura escolar y bullyng hipótesis sobre la relación violenciajuventud. Educación y ciudad, (18), 145-158.

Quevedo, F.J., y Rueda, E. P. (2006). Preguntas de madres y padres. Buenos Aires: Editorial Médica Panamericana.

Quintero, S., y Valencia, B. (2018). La didáctica en profesores de educación parvularia o preescolar: Los casos de Colombia y Chile. En Edgar Serna (Ed.) Revolución en la Formación y la Capacitación para el Siglo XXI (pp. 86-93). Medellín: Editorial Instituto Antioqueño de Investigación. Recuperado de https://www.researchgate.net/profile/Edgar_Serna_M/publication/331385401_Rev olucion_en_la_Formacion_y_la_Capacitacion_para_el_Siglo_XXI_ed_1/links/5c76ed d4458515831f756770/Revolucion-en-la-Formacion-y-la-Capacitacion-para-el-SigloXXI-ed-1.pdf\#page $=89$

Rodas, K. (2016). Los niños de edad escolar, en el conflicto del fenómeno del divorcio. (Tesis de Pregrado). Universidad de Cuenca, Cuenca, Ecuador. Recuperado de http://dspace.ucuenca.edu.ec/jspui/handle/123456789/25034

Valdivieso, Z. (2018). Estudio Filantrópico del Modelo Pedagógico de Praxeología de UNIMINUTO: Aproximación Analógica a Nivel Socio-Educativo. Educación y Humanismo, 20(34), 187-200. Doi: https://doi.org/10.17081/eduhum.20.34.2864 
Villao, O., y Espinoza, G. (2016).Trabajo autónomo y el desempeño académico en la carrera de educación básica. Revista Ciencias Pedagógicas e Innovación, 3(1) ,3438. Doi: https://doi.org/10.26423/rcpi.v3i1.11 